\title{
Modulation of ceramide metabolism in T-leukemia cell lines potentiates apoptosis induced by the cationic antimicrobial peptide bovine lactoferricin
}

\author{
SUZANNE J. FURLONG ${ }^{1}$, NEALE D. RIDGWAY ${ }^{2,3}$ and DAVID W. HOSKIN ${ }^{1,4}$ \\ Departments of ${ }^{1}$ Microbiology and Immunology, ${ }^{2}$ Pediatrics, ${ }^{3}$ Biochemistry and Molecular Biology \\ and ${ }^{4}$ Pathology, Faculty of Medicine, Dalhousie University, Halifax, Nova Scotia, B3H 1X5, Canada
}

Received September 3, 2007; Accepted October 30, 2007

\begin{abstract}
Bovine lactoferricin (LfcinB) is a cationic antimicrobial peptide that selectively induces apoptosis in several different types of human cancer cells. However, the potential use of LfcinB as an anticancer agent is presently limited by the need for relatively high concentrations of the peptide to trigger apoptosis. Ceramide is a membrane sphingolipid that is believed to function as a second messenger during apoptosis. In this study, we investigated the role of ceramide in LfcinBinduced apoptosis in CCRF-CEM and Jurkat T-leukemia cell lines. Exposure to LfcinB caused nuclear condensation and fragmentation, poly(ADP-ribose) polymerase (PARP) cleavage, and DNA fragmentation in CCRF-CEM and Jurkat T-cell acute lymphoblastic leukemia cell lines. Treatment with $\mathrm{C}_{6}$ ceramide, a cell-permeable, short-chain ceramide analog, also induced apoptotic nuclear morphology, PARP cleavage, and DNA fragmentation in T-leukemia cells. Although LfcinB treatment did not cause ceramide to accumulate in CCRF-CEM or Jurkat cells, the addition of $\mathrm{C}_{6}$ ceramide to LfcinB-treated T-leukemia cells resulted in increased DNA fragmentation. Furthermore, modulation of cellular ceramide metabolism either by inhibiting ceramidases with D-erythro-2-( $N$-myristoylamino)-1-phenyl-1-propanol or $N$-oleoylethanolamine, or by blocking glucosylceramide synthase activity with 1-phenyl-2-palmitoylamino-3-morpholino-1-propanol, enhanced the ability of LfcinB to trigger apoptosis in both Jurkat and CCRF-CEM cells. In addition, LfcinB-induced apoptosis of T-leukemia cells was enhanced in the presence of the antiestrogen tamoxifen, which has multiple effects on cancer cells, including inhibition of glucosylceramide
\end{abstract}

Correspondence to: Dr David Hoskin, Department of Microbiology and Immunology, Faculty of Medicine, Dalhousie University, Sir Charles Tupper Medical Building, 5850 College Street, Halifax, Nova Scotia, B3H 1X5, Canada

E-mail: d.w.hoskin@dal.ca

Key words: apoptosis, ceramide, ceramidase, glucosylceramide synthase, lactoferricin, leukemia synthase activity. We conclude that manipulation of cellular ceramide levels in combination with LfcinB therapy warrants further investigation as a novel strategy for the treatment of $\mathrm{T}$ cell-derived leukemias.

\section{Introduction}

Acute lymphoblastic leukemia (ALL) of T-cell or B-cell origin is the most common leukemia diagnosed in children and adolescents, whereas ALL occurs less frequently in adults (1). Fortunately, $70-83 \%$ of children with ALL can be cured by chemotherapy (2); recent improvements to treatment regimens suggest that a cure rate approaching $90 \%$ will soon be possible for childhood ALL (3). In contrast, only $24 \%$ of adults with ALL survive for 5 years following standard chemotherapy (4). Although the survival rate of adult ALL patients has improved with the advent of allogeneic hematopoietic stem cell transplantation (2), a substantial proportion of transplant recipients fail to achieve long-term disease-free survival (5). Novel therapeutic strategies are therefore needed for the treatment of ALL patients who cannot be cured by chemotherapy or allogeneic hematopoietic stem cell transplantation.

Bovine lactoferricin (LfcinB) is a cationic antimicrobial peptide consisting of 25 amino acids that is generated by acidpepsin hydrolysis of bovine lactoferrin (6). LfcinB possesses potent in vitro and in vivo anticancer activity. In vitro exposure to LfcinB causes apoptosis in several human cancer cell lines, including THP-1 human monocytic leukemia cells and MDA-MB-435 breast carcinoma cells, without having any cytotoxic effect on untransformed cells (7-9). We have recently demonstrated that LfcinB-induced apoptosis is initiated by the production of reactive oxygen species and the subsequent activation of caspase-2, which leads to mitochondrial membrane permeabilization, release of cytochrome $c$, and the sequential activation of caspase-9 and caspase-3 (8). Importantly, intratumoral injections of LfcinB inhibit the growth of murine Meth A fibrosarcoma, B16F10 melanoma, and C26 colon carcinoma cells in syngeneic mice (10), as well as human SH-SY-5Y neuroblastoma cells in nude rats (11). In addition, subcutaneous injections of LfcinB prevent the metastasis of murine L5178Y-ML25 lymphoma cells and B16-BL6 melanoma cells in syngeneic mice (12). Although 
LfcinB shows potential for use in the treatment of several human cancers, including leukemias, the feasibility of LfcinBbased therapy is at present limited because of the need to employ high concentrations (typically, $100-200 \mu \mathrm{g} / \mathrm{ml}$ in vitro) of the peptide in order to obtain an optimal cytotoxic effect.

Ceramide is a bioactive membrane sphingolipid implicated in apoptosis induced by diverse stimuli, including death receptor ligation, exposure to chemotherapeutic agents, ionizing radiation, and hypoxia $(13,14)$. Cellular ceramide accumulates prior to the onset of apoptosis as a result of de novo ceramide synthesis or the hydrolysis of sphingomyelin by activated sphingomyelinase. Although the mechanism(s) by which ceramide promotes apoptosis remain under investigation, ceramide is known to interact with a number of proteins involved in apoptosis induction, including protein phosphatase 2A (15), Raf-1 and extracellular signalregulated kinase (p44/p42) (16), and cathepsin D (17). In addition, ceramide treatment of $\mathrm{T}$ cell lines causes caspase-2dependent activation of caspase- 8 , followed by the loss of mitochondrial membrane potential, activation of caspase- 9 and caspase-3, and cell death by apoptosis (18). Recently, ceramide has been suggested to form channels in the outer membrane of the mitochondrion, leading to the release of proapoptotic mitochondrial proteins such as cytochrome $c$ (19). Interestingly, ceramide augments paclitaxel-mediated cytotoxicity in cultures of human leukemia cells and head and neck squamous carcinoma cells, suggesting that ceramide and chemotherapeutic agents may engage converging pathways during the induction of apoptosis $(20,21)$. Recently, we have shown that LfcinB-induced apoptosis of MDA-MB-435 breast carcinoma cells is enhanced in the presence of $\mathrm{C}_{6}$ ceramide, which is a cell-permeable, short-chain, analogue of ceramide (9). However, it is not known whether exposure to LfcinB itself causes cellular ceramide levels to increase in human leukemia cells.

On the basis that ceramide plays a central role in apoptosis, pharmacologic modulation of ceramide metabolism in order to cause the accumulation of cellular ceramide has been proposed as a novel strategy to increase the effectiveness of cancer chemotherapy $(22,23)$. Enzymes involved in ceramide metabolism include acidic and/or alkaline ceramidases and glucosylceramide synthase. Deacylation of ceramide by alkaline and acidic ceramidases produces sphingosine $(24,25)$, which can then be phosphorylated to yield sphingosine-1-phosphate, a molecule that inhibits ceramide-mediated apoptosis and promotes cell survival and proliferation $(26,27)$. Glucosylceramide synthase converts ceramide to glucosylceramide (28), thereby promoting cellular proliferation and preventing apoptosis (29). It is noteworthy that acidic ceramidase is overexpressed in prostate carcinoma cells (30), while overexpression of glucosylceramide synthase is associated with the development of chemoresistance in HL-60 promyelocytic leukemia and MCF-7 breast carcinoma cell lines $(31,32)$. Drugs that inhibit ceramidases or glucosylceramide synthase may therefore increase the effectiveness of apoptosis-inducing anticancer agents. However, the effect of ceramidase or glucosylceramide synthase inhibitors on LfcinB-induced apoptosis of human leukemia cells has not been determined.

In this study, we investigated the role of cellular ceramide in LfcinB-induced apoptosis of Jurkat and CCRF-CEM T- leukemia cells, which are cell lines derived from human acute lymphoblastic T-leukemia cells $(33,34)$. We also determined whether inhibition of ceramidases or glucosylceramide synthase involved in ceramide metabolism might increase the cytotoxic effect of LfcinB on T-leukemia cells. Our findings reveal that inhibition of ceramidases or glucosylceramide synthase is an effective strategy to enhance the apoptosisinducing activity of LfcinB, even though LfcinB by itself does not cause cellular ceramide to accumulate in $\mathrm{T}$ leukemia cells.

\section{Materials and methods}

Cell lines. CCRF-CEM T-leukemia cells were generously provided by Dr W. Gati (University of Alberta, Edmonton, Alberta, Canada). Jurkat T-leukemia cells were obtained from the American Type Culture Collection (Manassas, VA). Both cell lines were maintained in RPMI-1640 medium (SigmaAldrich Canada, Oakville, Ontario, Canada), supplemented with $100 \mu \mathrm{g} / \mathrm{ml}$ streptomycin, $100 \mathrm{U} / \mathrm{ml}$ penicillin, $2 \mathrm{mM}$ L-glutamine, $5 \mathrm{mM} N$-(2-hydroxyethyl)piperazine- $N$ '-(2ethanesulfonic acid) (HEPES) buffer ( $\mathrm{pH} 7.4)$ and $5 \%$ heat-inactivated fetal calf serum (FCS; all from Invitrogen, Burlington, Ontario, Canada) at $37^{\circ} \mathrm{C}$ in a $5 \% \mathrm{CO}_{2}$ humidified atmosphere. Cell cultures were passaged every second day or as required.

Reagents. LfcinB (amino acid sequence: FKCRRWQWRMK KLGAPSITCVRRAF) was synthesized in linear form with a purity of $>95 \%$ by Sigma Genosys (The Woodlands, TX). LfcinB was dissolved in serum-free RPMI-1640 medium and stored at $-80^{\circ} \mathrm{C}$. Hoescht 33342 trihydrochloride dye was from Sigma-Aldrich. $\mathrm{C}_{6}$ ceramide, $\mathrm{C}_{6}$ dihydroceramide, DL-threo-1phenyl-2-palmitoylamino-3-morpholino-1-propanol (PPMP), $N$-oleoylethanolamine (NOE), D-erythro-2-( $N$-myristoylamino)-1-phenyl-1-propanol (MAPP), and tamoxifen were from Biomol Research Laboratories Inc. (Plymouth Meeting, PA). Stock solutions of $\mathrm{C}_{6}$ ceramide, $\mathrm{C}_{6}$ dihydroceramide, MAPP, NOE, and tamoxifen were prepared in dimethyl sulfoxide and stored at $-20^{\circ} \mathrm{C}$. A $10-\mathrm{mM}$ stock solution of PPMP was prepared in water and stored at $-20^{\circ} \mathrm{C}$. Tritiatedthymidine $\left(\left[{ }^{3} \mathrm{H}\right] \mathrm{TdR}\right)$ and $\left[\gamma^{-32} \mathrm{P}\right]$-adenosine 5 '-triphosphate were obtained from MP Biomedicals (Irvine, CA) and Perkin-Elmer (Mississauga, Ontario, Canada], respectively. Anti-human poly(ADP-ribose) polymerase (PARP) monoclonal antibody $(\mathrm{mAb})$, anti-human actin $\mathrm{mAb}$, and horseradish peroxidase (HRP)-conjugated goat anti-mouse IgG were from Santa Cruz Biotechnology, Inc. (Santa Cruz, CA).

$\left[{ }^{3} \mathrm{H}\right] T d R$-release assay. DNA fragmentation was measured using a $\left[{ }^{3} \mathrm{H}\right] \mathrm{TdR}$-release assay (35). Briefly, T-leukemia cells were labeled with $\left[{ }^{3} \mathrm{H}\right] \mathrm{TdR}(5 \mu \mathrm{Ci} / \mathrm{ml})$ for $4 \mathrm{~h}$ at $37^{\circ} \mathrm{C}$ in a $5 \% \mathrm{CO}_{2}$ humidified atmosphere. Following 3 washes, radiolabeled cells were resuspended in RPMI-1640 medium and added in quadruplicate $\left(5 \times 10^{4}\right.$ cells/well) to 96 -well flatbottom tissue culture plates (Sarstedt Inc., St. Laurent, Quebec, Canada). All experiments were performed in RPMI-1640 medium containing $0.5 \%$ FCS since LfcinB has maximum 
cytotoxic activity at low serum concentrations (7). T-leukemia cells were cultured under the desired conditions for $12 \mathrm{~h}$ at $37^{\circ} \mathrm{C}$ in a $5 \% \mathrm{CO}_{2}$ humidified atmosphere. DNA was then harvested onto glass fiber filters using a multiple sample harvester (Skatron Instruments, Sterling, VA) and radioactivity in counts per minute (cpm) was measured by liquid scintillation counting. Percent DNA fragmentation was calculated by the formula $\left[\left(\mathrm{C}_{\mathrm{cpm}}-\mathrm{E}_{\mathrm{cpm}}\right) / \mathrm{C}_{\mathrm{cpm}}\right] \times 100$, where $\mathrm{E}$ is intact DNA from treated cells and $\mathrm{C}$ is intact DNA from control cells.

Hoechst staining. T-leukemia cells with apoptotic nuclear morphology were distinguished from normal cells by staining with DNA-specific Hoescht 33342 trihydrochloride dye (36). T-leukemia cells were resuspended in RPMI-1640 medium containing $0.5 \%$ FCS and added in duplicate $\left(5 \times 10^{5}\right.$ cells/well $)$ to 24-well flat-bottom plates (Sarstedt Inc.). T-leukemia cells were cultured under the desired conditions for $12 \mathrm{~h}$ at $37^{\circ} \mathrm{C}$ in a $5 \% \mathrm{CO}_{2}$ humidified atmosphere, then harvested and resuspended in a $4 \%$ paraformaldehyde solution. Aliquots of T-leukemia cells were placed onto silinated slides, air-dried, and stained with $10 \mu \mathrm{g} / \mathrm{ml}$ Hoecsht 33342 dye for $10 \mathrm{~min}$. Slides were then washed with phosphate-buffered saline (PBS) and coverslips were mounted with $10 \%$ glycerol/ phosphate-buffered saline solution. Nuclear condensation and fragmentation were visualized by fluorescence microscopy.

Detection of PARP cleavage. Western blotting was used to measure PARP cleavage, which is an early marker of apoptosis (37). T-leukemia cells in RPMI-1640 medium containing $0.5 \%$ FCS were cultured in the absence or presence of LfcinB $(50 \mu \mathrm{g} / \mathrm{ml})$ or $\mathrm{C}_{6}$ ceramide $(50 \mu \mathrm{M})$ for 6 and $12 \mathrm{~h}$. Cells were then lysed in ice-cold lysis buffer $[50 \mathrm{mM}$ Tris- $\mathrm{HCl}$ at $\mathrm{pH} 7.5,150 \mathrm{mM} \mathrm{NaCl}, 50 \mathrm{mM} \mathrm{Na} 2 \mathrm{HPO}_{4}, 0.25 \%$ sodium deoxycholate (w/v), 0.1\% Nonidet P-40 (v/v), $1 \mathrm{mM} \mathrm{Na}_{3} \mathrm{VO}_{4}$, $1 \mathrm{mM} \mathrm{NaF}, 5 \mathrm{mM}$ ethylenediaminetetraacetic acid (EDTA), and $5 \mathrm{mM}$ ethylene glycol-bis(ß-aminoethyl ether)-N,N,N',N'tetraacetic acid] containing freshly-added protease inhibitors (1 $\mathrm{mM}$ phenylmethylsulfonyl fluoride, $10 \mu \mathrm{g} / \mathrm{ml}$ aprotinin, $10 \mu \mathrm{g} / \mathrm{ml}$ leupeptin and $10 \mu \mathrm{g} / \mathrm{ml}$ pepstatin). The protein content of cell lysates was determined by Bradford assay (Bio-Rad Laboratories Ltd., Mississauga, Ontario, Canada). T-leukemia cell lysates $(5 \mu \mathrm{g})$ were resolved by electrophoresis across a $10 \%$ SDS polyacrylamide gel. Protein bands were transferred to nitrocellulose membranes and the resulting blots were blocked overnight with PBS-Tween-20 (0.25 M Tris at $\mathrm{pH} 7.5,150 \mathrm{mM} \mathrm{NaCl}, 0.2 \%$ Tween-20 in PBS) containing 5\% powdered skim milk (w/v). Blots were probed overnight with anti-PARP mAb (1:500), then washed with PBS-Tween-20 and probed for $1 \mathrm{~h}$ with HRP-conjugated goat anti-mouse IgG antibody $(10 \mu \mathrm{g} / \mathrm{ml})$. After additional washes with PBS-Tween 20, the protein bands were visualized using an enhanced chemiluminescence detection system (Bio-Rad Laboratories). Blots were stripped and re-probed with antiactin $\mathrm{mAb}(1: 1000)$ to confirm equal protein loading.

Measurement of cellular ceramide. T-leukemia cells were resuspended in RPMI-1640 medium containing 0.5\% FCS and added in triplicate $\left(5 \times 10^{5}\right.$ cells/well) to 24 -well flat-bottom plates. T-leukemia cells were then cultured in the absence or presence of $\mathrm{LfcinB}$ for $2-12 \mathrm{~h}$ at $37^{\circ} \mathrm{C}$ in a $5 \% \mathrm{CO}_{2}$ humidified atmosphere. T-leukemia cells were harvested and resuspended in 5:4 methanol/water (v/v). Protein concentration was determined using the Bradford protein assay (Bio-Rad Laboratories). Lipids were extracted by adding 1:2 chloroform/ methanol (v/v) and $0.58 \% \mathrm{NaCl}$, followed by centrifugation at $400 \mathrm{~g}$ for $5 \mathrm{~min}$ to separate phases. The upper aqueous phase was removed and the bottom phase was washed twice with 45:47:5 methanol/0.58\% NaCl/chloroform (v/v). The bottom phase was then dryed under nitrogen, and the lipids were dissolved in chloroform. Ceramide mass in the total lipid extract was then determined by the bacterial diglyceride kinase assay (38). Briefly, the lipids were solubilized in a $7.5 \%$ octyl- $\beta-\mathrm{D}$-glucoside $/ 5 \mathrm{mM}$ cardiolipin solution by sonication. Reaction buffer (100 mM imidazole $\mathrm{HCl}, \mathrm{pH}$ 6.6, $100 \mathrm{mM} \mathrm{NaCl}, 25 \mathrm{mM} \mathrm{MgCl}$, and $2 \mathrm{mM}$ EGTA), $100 \mathrm{mM}$ dithiothreitol, and diluted diglyceride kinase membranes (EMD Biosciences Inc., San Diego, CA) were added to the solubilized lipids. The reaction was then initiated by the addition of $10 \mathrm{mM}\left[\gamma^{3}{ }^{32} \mathrm{P}\right]$-adenosine 5'-triphosphate and was terminated by adding 1:2 choloroform/methanol $(\mathrm{v} / \mathrm{v})$ and $1 \% \mathrm{HClO}_{4}$ after incubation at $25^{\circ} \mathrm{C}$ for $45 \mathrm{~min}$. Lipids were extracted and the lower chloroform phase was dried under nitrogen. Lipids were dissolved in 2:1 chloroform/methanol $(\mathrm{v} / \mathrm{v})$ and spotted onto a thin layer chromatography plate (silca gel 60), which was then developed using 65:15:5 chloroform/methanol/acetic acid (v/v). Phosphorylated lipids were visualized by radioautography and the area corresponding to phosphorylated ceramide was scraped off and radioactivity was measured by liquid scintillation counting. Total ceramide mass from the original sample was determined using a standard curve established using known concentrations of ceramide.

Statistical analysis. Data were analyzed using the Instat statistics program (GraphPad Software Inc., San Diego, CA). Statistical comparisons were performed using one-way analysis of variance (ANOVA) and the Tukey-Kramer multiple comparisons test.

\section{Results}

LfcinB induces apoptosis in Jurkat and CCRF-CEM Tleukemia cells. Fig. 1A shows that culture for $12 \mathrm{~h}$ in the presence of 25,50 , or $100 \mu \mathrm{g} / \mathrm{ml} \mathrm{LfcinB}$ caused dose-dependent DNA fragmentation in Jurkat and CCRF-CEM T-leukemia cell lines. A subsequent kinetics analysis indicated that LfcinB-induced DNA fragmentation in Jurkat and CCRFCEM cells was maximal at the $12 \mathrm{~h}$ time-point (data not shown). To confirm that LfcinB-induced DNA fragmentation in T-leukemia cells was the result of apoptosis, Jurkat and CCRF-CEM cells were cultured in the absence or presence of $50 \mu \mathrm{g} / \mathrm{ml} \mathrm{LfcinB}$ for $12 \mathrm{~h}$, and then stained with DNAspecific Hoechst 33342 dye. Alternately, T-leukemia cells were cultured in the absence or presence of $50 \mu \mathrm{g} / \mathrm{ml} \mathrm{LfcinB}$ for 6 or $12 \mathrm{~h}$, after which PARP cleavage was measured by Western blotting. LfcinB treatment of Jurkat and CCRF-CEM cells resulted in nuclear condensation and fragmentation (Fig. 1B), as well as PARP cleavage (Fig. 1C), indicating that LfcinB-induced cell death was by apoptosis. 

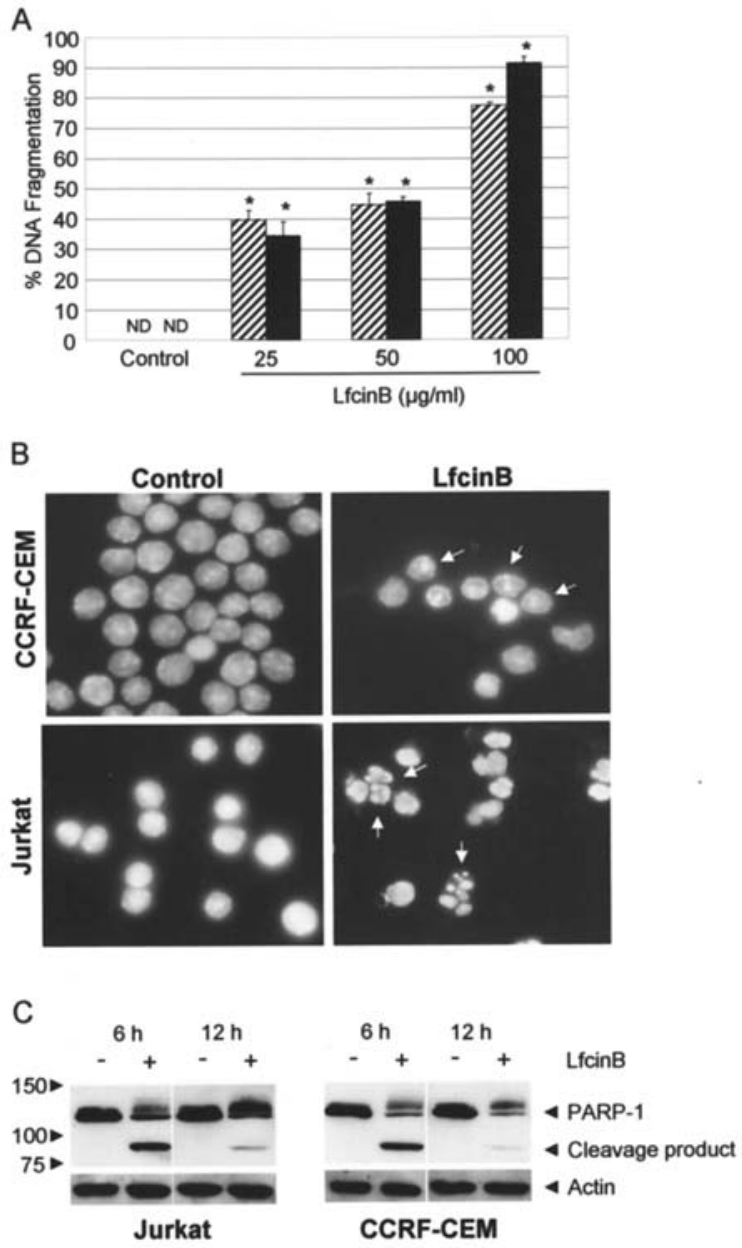

Figure 1. LfcinB triggers apoptosis in T-leukemia cells. (A) Jurkat (hatched) or CCRF-CEM (solid) T-leukemia cells were cultured in the absence (vehicle only) or presence of the indicated concentrations of LfcinB for $12 \mathrm{~h}$. DNA fragmentation was then measured by $\left[{ }^{3} \mathrm{H}\right] \mathrm{TdR}$-release assay. Data from a representative experiment $(\mathrm{n}=3)$ are expressed as \% DNA fragmentation $\pm \mathrm{SD} .{ }^{*} \mathrm{P}<0.001$ in comparison to the medium control by the Tukey-Kramer multiple comparisons test. (B) Jurkat or CCRF-CEM cells were cultured in the absence (vehicle only) or presence of $50 \mu \mathrm{g} / \mathrm{ml} \mathrm{LfcinB}$ for $12 \mathrm{~h}$. Cells were then fixed, stained with DNA-specific Hoescht 33342 dye, and visualized by fluorescence microscopy. Intense staining indicates nuclear condensation. Arrows indicate cells showing nuclear fragmentation. (C) Jurkat or CCRFCEM cells were cultured in the absence (vehicle only) or presence of $50 \mu \mathrm{g} / \mathrm{ml}$ LfcinB for 6 or $12 \mathrm{~h}$. Cell lysates were then prepared and subjected to Western blot analysis for the detection of PARP cleavage. Blots were stripped and re-probed for actin expression to confirm equal protein loading.

LfcinB-induced apoptosis is not associated with the accumulation of cellular ceramide. We next compared cellular ceramide levels in control and LfcinB-treated Jurkat and CCRF-CEM T-leukemia cells because ceramide has been identified as an important second messenger during apoptosis $(13,14)$. Ceramide measurements were performed $2,4,8$, and $12 \mathrm{~h}$ after exposing T-leukemia cells to $50 \mu \mathrm{g} / \mathrm{ml} \mathrm{LfcinB}$. Fig. 2 shows that control and LfcinB-treated Jurkat and CCRF-CEM cells had equivalent cellular ceramide content, indicating that LfcinB treatment did not cause cellular ceramide levels to become elevated in T-leukemia cells.

$C_{6}$ ceramide induces apoptosis in Jurkat and CCRF-CEM T-leukemia cells. To determine whether T-leukemia cells were sensitive to the cytotoxic effect of cellular ceramide

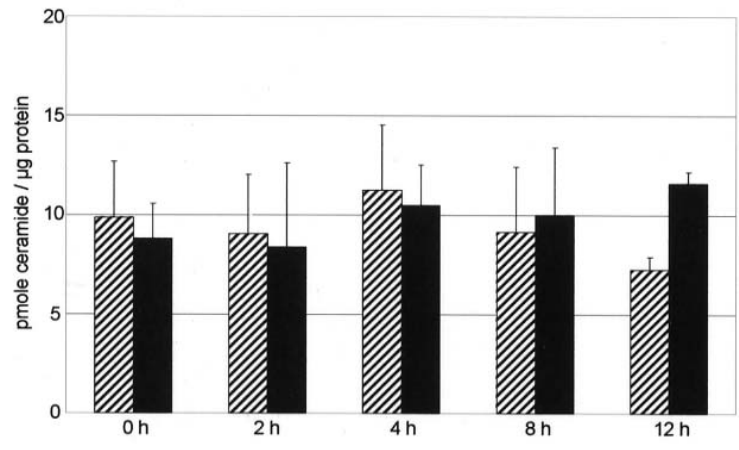

Figure 2. LfcinB does not induce ceramide accumulation in T-leukemia cells. Jurkat (hatched) or CCRF-CEM (solid) T-leukemia cells were cultured for the indicated times in the absence (vehicle only) or presence of $50 \mu \mathrm{g} / \mathrm{ml} \mathrm{LfcinB}$. Lipids were extracted from harvested cells and ceramide mass was determined as described in Materials and methods. Data are the mean of three independent experiments \pm SEM

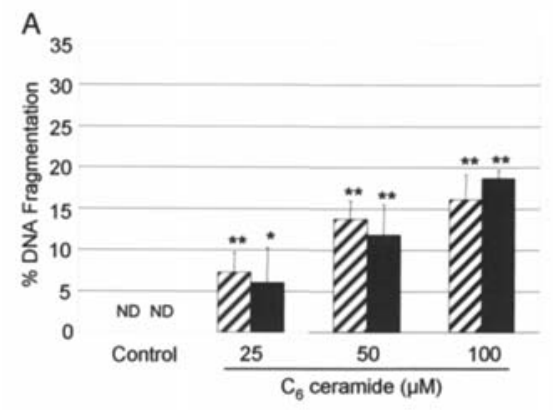

B

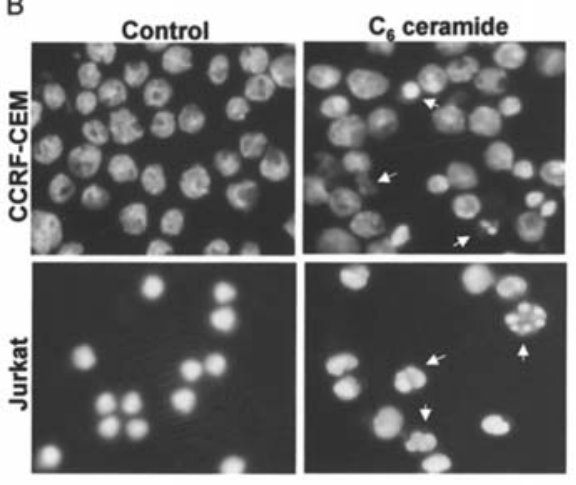

C

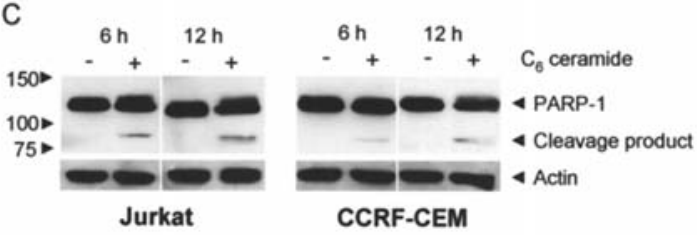

Figure 3. $\mathrm{C}_{6}$ ceramide triggers apoptosis in T-leukemia cells. (A) Jurkat (hatched) or CCRF-CEM (solid) T-leukemia cells were cultured in the absence (vehicle only) or presence of the indicated concentrations of $\mathrm{C}_{6}$ ceramide for $12 \mathrm{~h}$. DNA fragmentation was then measured by $\left[{ }^{3} \mathrm{H}\right] \mathrm{TdR}$-release assay. Data from a representative experiment $(\mathrm{n}=3)$ are expressed as \% DNA fragmentation $\pm \mathrm{SD} .{ }^{*} \mathrm{P}<0.01 ;{ }^{* *} \mathrm{P}<0.001$ in comparison to the vehicle control by the Tukey-Kramer multiple comparisons test. (B) Jurkat or CCRF-CEM cells were cultured in the absence (vehicle only) or presence of $100 \mu \mathrm{M}$ $\mathrm{C}_{6}$ ceramide for $12 \mathrm{~h}$. Cells were then fixed, stained with DNA-specific Hoescht 33342 dye, and visualized by fluorescence microscopy. Intense staining indicates nuclear condensation. Arrows indicate cells showing nuclear fragmentation. (C) Jurkat or CCRF-CEM cells were cultured in the absence (vehicle only) or presence of $50 \mu \mathrm{M} \mathrm{C}_{6}$ ceramide for 6 or $12 \mathrm{~h}$. Cell lysates were then prepared and subjected to Western blot analysis for the detection of PARP cleavage. 
A

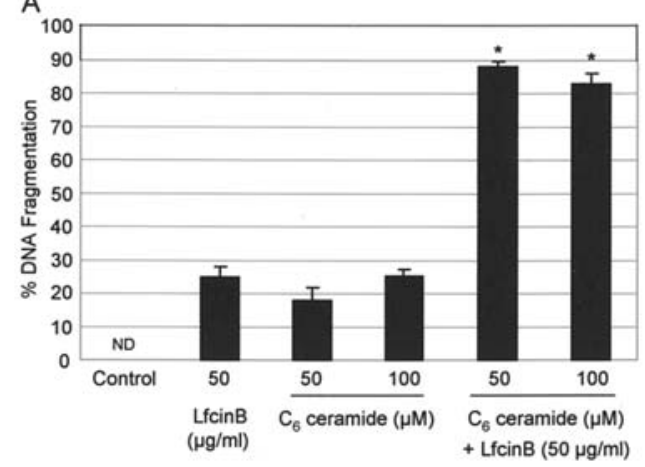

B

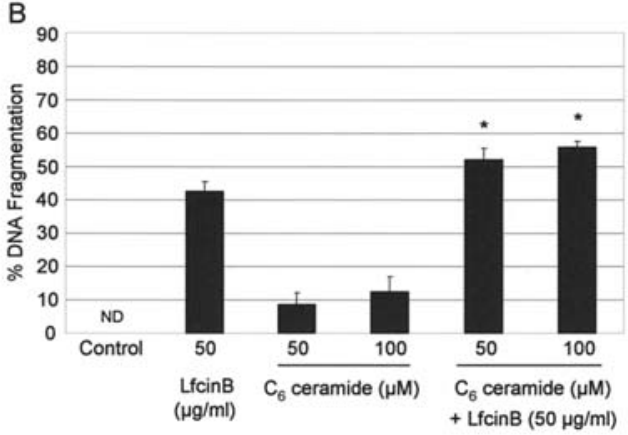

Figure 4. $\mathrm{C}_{6}$ ceramide enhances LfcinB-induced DNA fragmentation in T-leukemia cells. (A) Jurkat or (B) CCRF-CEM T-leukemia cells were cultured for $12 \mathrm{~h}$ in the absence (vehicle only) or presence of LfcinB and/or $\mathrm{C}_{6}$ ceramide at the indicated concentrations. DNA fragmentation was then measured by $\left[{ }^{3} \mathrm{H}\right] \mathrm{TdR}$-release assay. Data from a representative experiment $(\mathrm{n}=3)$ are expressed as \% DNA fragmentation $\pm \mathrm{SD} .{ }^{*} \mathrm{P}<0.001$ in comparison to cells treated with LfcinB or $\mathrm{C}_{6}$ ceramide alone by the Tukey-Kramer multiple comparisons test.

accumulation, Jurkat and CCRF-CEM cells were cultured for $12 \mathrm{~h}$ in the absence or presence of a 25,50 , or $100 \mu \mathrm{M}$ concentration of $\mathrm{C}_{6}$ ceramide, which is a cell-permeable, short-chain analog of ceramide. DNA fragmentation was then measured by $\left[{ }^{3} \mathrm{H}\right] \mathrm{TdR}$-release assay. The intracellular accumulation of cell-permeable ceramide analogs mimics the apoptosis-inducing effect of elevated cellular ceramide (39). Fig. 3A shows that exposure to $\mathrm{C}_{6}$ ceramide caused dose-dependent DNA fragmentation in both T-leukemia cell lines. In addition, nuclear condensation and fragmentation (Fig. 3B), as well as PARP cleavage (Fig. 3C) were observed in $\mathrm{C}_{6}$ ceramide-treated Jurkat and CCRF-CEM cells, indicating that exposure to $\mathrm{C}_{6}$ ceramide caused T-leukemia cells to die by apoptosis. In contrast, the viability of Jurkat and CCRF-CEM cells was not reduced following treatment with a 25,50 , or $100 \mu \mathrm{M}$ concentration of $\mathrm{C}_{6}$ dihydroceramide, which is an inactive analog of $\mathrm{C}_{6}$ ceramide (data not shown).

Combination treatment with $L f \operatorname{cin} B$ and $C_{6}$ ceramide leads to increased cytotoxicity. Since T-leukemia cells were sensitive to Lfcin $\mathrm{B}$ and $\mathrm{C}_{6}$ ceramide individually, we decided to investigate the effect of combined LfcinB and $\mathrm{C}_{6}$ ceramide treatment on cell viability. Fig. 4 shows that combined treatment with $\mathrm{LfcinB}$ and $\mathrm{C}_{6}$ ceramide had a greater cytotoxic effect on Jurkat and CCRF-CEM cells than was seen when either agent was used alone. Jurkat cells showed a synergistic increase in DNA fragmentation following exposure to

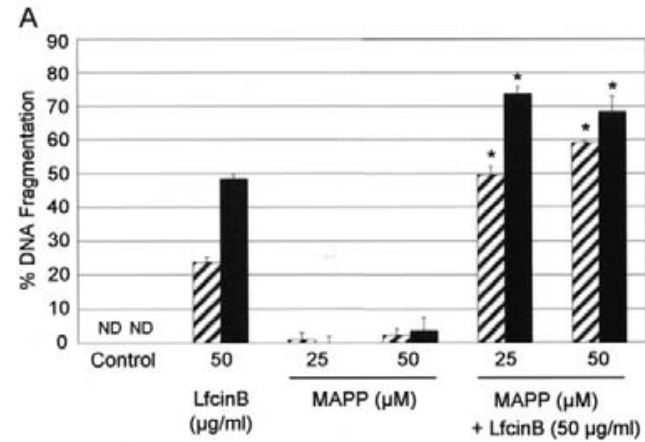

B

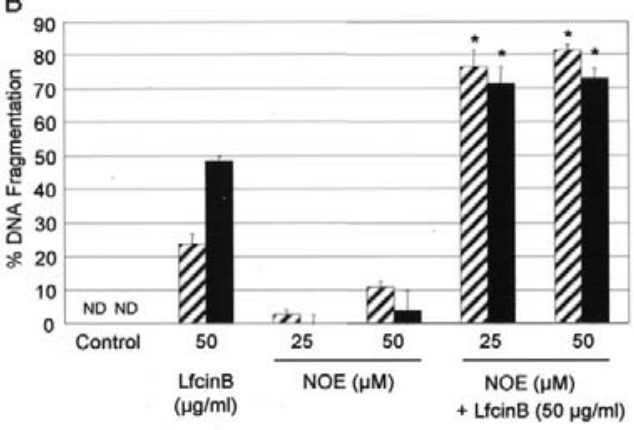

Figure 5. Ceramidase inhibitors enhance LfcinB-induced DNA fragmentation in T-leukemia cells. Jurkat (hatched) or CCRF-CEM (solid) T-leukemia cells were cultured for $12 \mathrm{~h}$ in the absence (vehicle only) or presence LfcinB and/or (A) MAPP or (B) NOE at the indicated concentrations. DNA fragmentation was then measured by $\left[{ }^{3} \mathrm{H}\right] \mathrm{TdR}$-release assay. Data from a representative experiment $(n=3)$ are expressed as \% DNA fragmentation $\pm \mathrm{SD} .{ }^{*} \mathrm{P}<0.001$ in comparison to the single agent-treated cells by the TukeyKramer multiple comparisons test.

combined LfcinB and $\mathrm{C}_{6}$ ceramide (Fig. 4A) whereas CCRF-CEM cells exhibited an additive increase in DNA fragmentation when cultured in the presence of combined LfcinB and $\mathrm{C}_{6}$ ceramide (Fig. 4B).

Inhibition of ceramidases enhances the cytotoxic effect of LfcinB. Since combination treatment with LfcinB and cellpermeable $\mathrm{C}_{6}$ ceramide had a greater apoptosis-inducing effect than either agent alone, we hypothesized that inhibition of enzymes that normally prevent the accumulation of cellular ceramide might enhance LfcinB-induced T-leukemia cell death. Fig. 5A shows that concomitant treatment of Jurkat and CCRF-CEM cells with LfcinB and the acidic ceramidase inhibitor MAPP (25) resulted in significantly greater DNA fragmentation $(\mathrm{P}<0.001)$ than was evident following treatment with LfcinB alone. Fig. 5B shows that a significant increase $(\mathrm{P}<0.001)$ in DNA fragmentation over that caused by LfcinB alone was also observed when Jurkat and CCRF-CEM cells were cultured in the presence of LfcinB plus the alkaline ceramidase inhibitor NOE (24). Neither MAPP nor NOE alone had any substantial cytotoxic effect on Jurkat or CCRF-CEM cells. These data indicated that inhibition of acidic or alkaline ceramidases within T-leukemia cells enhanced the apoptosisinducing activity of LfcinB.

Inhibition of glucosylceramide synthase increases the cytotoxic effect of $L f \operatorname{cin} B$. We also cultured T-leukemia cells in the absence or presence of LfcinB, with or without the glucosyl- 


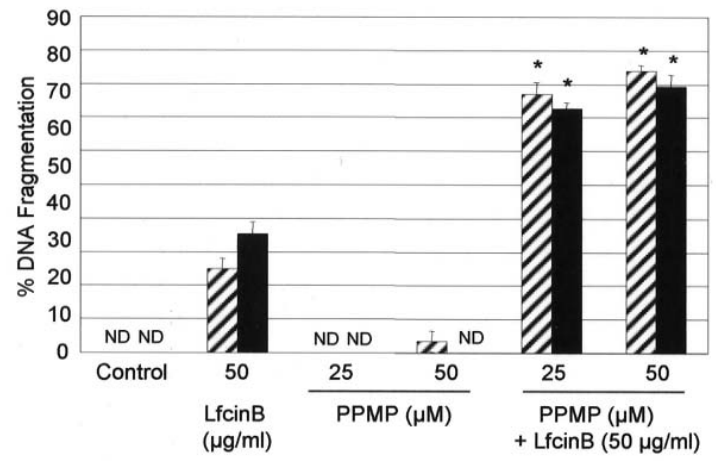

Figure 6. Glucosylceramide synthase inhibition enhances LfcinB-induced DNA fragmentation in T-leukemia cells. Jurkat (hatched) or CCRF-CEM (solid) T-leukemia cells were cultured for $12 \mathrm{~h}$ in the absence (vehicle only) or presence LfcinB and/or PPMP at the indicated concentrations. DNA fragmentation was then measured by $\left[{ }^{3} \mathrm{H}\right] \mathrm{TdR}$-release assay. Data from a representative experiment $(\mathrm{n}=3)$ are expressed as \% DNA fragmentation $\pm \mathrm{SD}$. ${ }^{*} \mathrm{P}<0.001$ in comparison to the single agent-treated cells by the Tukey-Kramer multiple comparisons test.

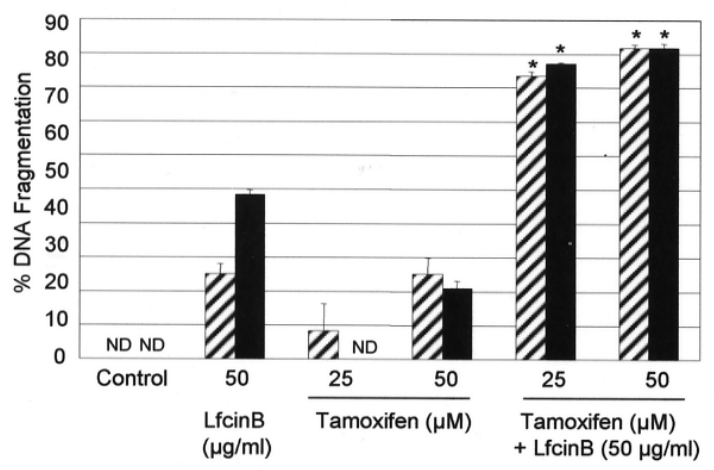

Figure 7. Tamoxifen enhances LfcinB-induced DNA fragmentation in Tleukemia cells. Jurkat (hatched) or CCRF-CEM (solid) T-leukemia cells were cultured for $12 \mathrm{~h}$ in the absence (vehicle only) or presence LfcinB and/or tamoxifen at the indicated concentrations. DNA fragmentation was then measured by $\left[{ }^{3} \mathrm{H}\right] \mathrm{TdR}$-release assay. Data from a representative experiment $(\mathrm{n}=3)$ are expressed as \% DNA fragmentation $\pm \mathrm{SD} .{ }^{*} \mathrm{P}<0.001$ in comparison to the single agent-treated cells by the Tukey-Kramer multiple comparisons test.

ceramide synthase inhibitor PPMP to prevent the conversion of endogenous ceramide to glucosylceramide (28). Fig. 6 shows that combination treatment with LfcinB and PPMP was far more effective than LfcinB alone in causing Jurkat and CCRF-CEM cells to die by apoptosis. Treatment with PPMP alone did not have any significant cytotoxic effect on Jurkat or CCRF-CEM cells. Interestingly, similar results were obtained when Jurkat and CCRF-CEM cells were exposed to LfcinB in combination with tamoxifen (Fig. 7), which has multiple apoptosis-promoting effects on cancer cells (40), including inhibition of ceramide glucosylation (41). Tamoxifen by itself had a modest cytotoxic effect on T-leukemia cells when used at the highest concentration tested $(50 \mu \mathrm{M})$. Taken together, these data indicated that blockade of glucosylceramide synthase activity within Tleukemia cells enhanced the apoptosis-inducing activity of LfcinB.

\section{Discussion}

We have shown potent induction of apoptosis, as indicated by DNA fragmentation, nuclear condensation and fragmentation, and PARP cleavage, following LfcinB treatment of two different T-leukemia cell lines. However, LfcinB-induced apoptosis was not associated with increased cellular ceramide content, which was in contrast to numerous reports that have implicated ceramide as an important second messenger during apoptosis caused by death receptor ligation or cellular stress due to exposure to chemotherapeutic agents or ionizing radiation $(13,14)$. This apparent discrepancy might be explained by our recent finding that LfcinB causes limited damage the cytoplasmic membrane of T-leukemia cells, leading to LfcinB uptake without any evidence of necrosis (42). LfcinB that gains access to the cytoplasmic compartment colocalizes with mitochondria, resulting in mitochondrial membrane permeabilization, the release of cytochrome $c$, and apoptosis induction. The ability of LfcinB to trigger apoptosis by direct disruption of mitochondrial membrane integrity might circumvent any need for apoptotic signaling via the ceramide pathway. Although T-leukemia cells failed to accumulate cellular ceramide in response to LfcinB treatment, both Jurkat and CCRF-CRM cells were sensitive to the cytotoxic effect of elevated cellular ceramide since exposure of these T-leukemia cell lines to cell-permeable $\mathrm{C}_{6}$ ceramide resulted in DNA fragmentation, PARP cleavage, and the appearance of nuclear morphology that was consistent with apoptosis. The intracellular accumulation of $\mathrm{C}_{6}$ ceramide has an apoptosisinducing effect comparable to that of an increased level of endogenous ceramide (39). Ceramide-induced apoptosis is known to involve the sequential activation of caspase- 2 and -8 , leading to mitochondrial membrane permeabilization and the activation of caspase-9 and caspase-3 (18). In addition, recent evidence suggests that ceramide is able to directly cause the loss of mitochondrial membrane integrity (19). However, the ability of ceramide analogues to trigger apoptosis may vary depending on the cellular context since $\mathrm{C}_{2}$ ceramide ( $N$-acetyl-D-erythro-sphingosine) induces apoptosis in HL-60 promyelocytic leukemia cells but causes B-chronic lymphocytic leukemia cells to die by a non-apoptotic mechanism (43).

Although LfcinB shows potential as a novel cytotoxic agent for the treatment of several human cancers, the fact that relatively high concentrations of LfcinB are required for optimal induction of cancer cell apoptosis may place limits on its therapeutic utility. The finding that LfcinB can be combined with $\mathrm{C}_{6}$ ceramide to create a greater apoptosisinducing effect than that of either agent alone on T-leukemia cells suggested that the mechanism of LfcinB-induced apoptosis was convergent with the apoptotic pathway triggered by the accumulation of cellular ceramide. Combination treatment with LfcinB and drugs that cause cellular ceramide to accumulate through the inhibition of endogenous ceramide metabolism might therefore be a feasible strategy to improve the anticancer activity of LfcinB. In this regard, we show here for the first time that LfcinB-induced apoptosis of T-leukemia cells was enhanced when acidic or alkaline ceramidasemediated ceramide metabolism was blocked with MAPP or NOE, respectively, or ceramide glucosylation was inhibited 
by PPMP; even though LfcinB-induced apoptosis in Jurkat and CCRF-CEM cells was not associated with the accumulation of cellular ceramide. It is possible that ceramidase and glucosylceramide synthase activity were heightened in T-leukemia cells in response to mitochondrial damage caused by LfcinB, resulting in increased sensitivity of T-leukemia cells to agents that block these pathways of ceramide metabolism. Our findings are in line with reports that killing of solid tumor cell lines by the synthetic retinoid N-(4-hydroxyphenyl) retinamide, which, like LfcinB (8), promotes mitochondrial membrane permeabilization and the release of cytochrome $c$ (44), is also enhanced in the presence of agents that inhibit ceramidase or glucosylceramide synthase function $(45,46)$. Elevated cellular ceramide levels caused by ceramidase or glucosylceramide synthase inhibition in LfcinB-treated Tleukemia cells may lead to a further loss of mitochondrial membrane integrity and increase in cytochrome $c$-dependent apoptosome formation, thereby augmenting LfcinB-induced apoptosis. Inhibition of acidic or alkaline ceramidases by MAPP or NOE, respectively, or glucosylceramide synthase by PPMP in the absence of LfcinB did not have any substantial cytotoxic effect on Jurkat or CCRF-CEM cells, suggesting that ceramidase-mediated deacylation of ceramide to sphingosine and glucosylceramide synthase-mediated conversion of ceramide to glucosylceramide are not normally major pathways of cellular ceramide metabolism in T-leukemia cells. Baseline ceramide metabolism within T-leukemia cells and other types of cancer cells is apparently different since inhibition of acidic ceramidase by NOE elevates cellular ceramide levels and increases apoptosis in prostate cancer cells (47) while inhibition of glucosylceramide synthase by PPMP causes cellular ceramide accumulation and apoptosis in colon carcinoma cells (48).

In addition to ceramide metabolism by ceramidases and glucosylceramide synthase, cellular ceramide can be reversibly converted to sphingomyelin by sphingomyelin synthase (49), acylated by $1-O$-acylceramide synthase to form $1-O$-acylceramide (50), or phosphorylated by ceramide kinase to yield ceramide-1-phosphate (51). Although there is at present no information on 1-O-acylceramide levels in human cancer cells, ceramide kinase activity has been detected in human HL-60 promyelocytic leukemia cells (51). In addition, inhibition of sphingomyelin synthase activity by tricyclodecan9-yl-xanthogenate (D609) causes human U937 monocytic leukemia cells to die by apoptosis via a mechanism that is associated with a substantial increase in cellular ceramide content (52). It is therefore reasonable to speculate that ceramide kinase and/or sphingomyelin synthase inhibitors might be combined with ceramidase and glucosylceramide synthase inhibitors to further enhance LfcinB-induced apoptosis in human T-leukemia cells.

It is noteworthy that LfcinB-induced apoptosis in Jurkat and CCRF-CEM cells was also enhanced in the presence of the antiestrogen tamoxifen, which mediates its anticancer activity via multiple mechanisms (40), including inhibition of ceramide glucosylation (41). We also observed that a $50 \mu \mathrm{M}$ concentration of tamoxifen alone had a modest apoptosisinducing effect on T-leukemia cells. Tamoxifen-induced cytotoxicity was most likely not related to the inhibitory effect of tamoxifen on glucosylceramide synthase since the glycosyl- ceramide synthase inhibitor PPMP by itself did not induce apoptosis in Jurkat or CCRF-CEM cells. In addition to inhibiting ceramide glucosylation, tamoxifen has been reported to trigger apoptosis by mechanisms that include Fas death receptor signaling (53), c-myc overexpression (54), and oxidative stress-related activation of c-Jun $\mathrm{NH}_{2}$-terminal kinase (55). Additional study is required to determine which, if any, of these mechanisms contribute to the ability of tamoxifen to enhance the cytotoxic effect of LfcinB on T-leukemia cells.

\section{Acknowledgements}

This work was funded by grants from the Natural Sciences and Engineering Research Council of Canada (NSERC), the Dairy Farmers of Canada, and the Leukemia and Lymphoma Society of Canada. S. Furlong was supported by a Research Trainee Award from the Cancer Research Training Program (with funding from the Dalhousie Cancer Research Program) and an NSERC Postgraduate Studentship.

\section{References}

1. Pui C-H and Evans WE: Acute lymphoblastic leukemia. N Engl J Med 339: 605-615, 1998.

2. Pui C-H and Evans WE: Treatment of acute lymphoblastic leukemia. N Engl J Med 354: 166-178, 2006.

3. Pui CH, Relling MV, Sandlund JT, Downing JR, Campana D and Evans WE: Rationale and design of Total Therapy Study XV for newly diagnosed childhood acute lymphoblastic leukemia. Ann Hematol 83 (Suppl. 1): S124-S126, 2004.

4. Thomas X, Danaila C, Le QH, Sebban C, Troncy J, Charrin C, Lheritier V, Michallet M, Magaud JP and Fiere D: Long-term follow-up of patients with newly diagnosed adult acute lymphoblastic leukemia: a single institution experience of 378 consecutive patients over a 21-year period. Leukemia 15: 1811-1822, 2001.

5. Thomas X, Boiron JM, Huguet F, Dombret H, Bradstock K, Vey N, Kovacsovics T, Delannoy A, Fegueux N, Fenaux P, Stamatoullas A, Vernant JP, Tournilhac O, Buzyn A, Reman O, Charrin C, Boucheix C, Gabert J, Lheriier V and Fiere D: Outcome of treatment in adults with acute lymphoblastic leukemia: analysis of the LALA-94 trial. J Clin Oncol 22: 4075-4086, 2004.

6. Bellamy W, Takase M, Wakabayashi H, Kawase K and Tomita M: Antibacterial spectrum of lactoferricin B, a potent bactericidal peptide derived from the $\mathrm{N}$-terminal region of bovine lactoferrin. J Appl Bacteriol 73: 72-79, 1992.

7. Yoo Y-C, Watanabe R, Koike Y, Mitobe M, Shimazaki K, Watanabe S and Azuma I: Apoptosis in human leukemic cells induced by lactoferricin, a bovine milk protein-derived peptide: involvement of reactive oxygen species. Biochem Biophys Res Commun 237: 624-628, 1997.

8. Mader JS, Salsman J, Conrad DM and Hoskin DW: Bovine lactoferricin selectively induces apoptosis in human leukemia and carcinoma cell lines. Mol Cancer Ther 4: 1-13, 2005.

9. Furlong SJ, Mader JS and Hoskin DW: Lactoferricin-induced apoptosis in estrogen-non-responsive MDA-MB-435 breast cancer cells is enhanced by C6 ceramide or tamoxifen. Oncol Rep 15: 1385-1390, 2006.

10. Eliassen LT, Berge G, Sveinbjørnsson B, Svendsen JS, Vorland LH and Rekdal $\varnothing$ : Evidence for a direct antitumor mechanism of action of bovine lactoferricin. Anticancer Res 22: 2703-2710, 2002.

11. Eliassen LT, Berge G, Leknessund A, Wikman M, Lindin I, Løkke C, Ponthan F, Johnsen JI, Sveinbjørnsson B, Kogner P, Flaegstad T and Rekdal $\varnothing$ : The antimicrobial peptide, lactoferricin $\mathrm{B}$, is cytotoxic to neuroblastoma cells in vitro and inhibits xenograft growth in vivo. Int J Cancer 119: 493-500, 2006.

12. Yoo YC, Watanabe S, Watanabe R, Hata K, Shimazaki K and Azuma I: Bovine lactoferrin and lactoferricin, a peptide derived from bovine lactoferrin, inhibit tumor metastasis in mice. Jpn J Cancer Res 88: 184-190, 1997.

13. Dbaibo GS and Hannun YA: Signal transduction and the regulation of apoptosis: roles of ceramide. Apoptosis 3: 317-334, 1998. 
14. Pettus BJ, Chalfant CE and Hannun YA: Ceramide in apoptosis: an overview and current perspectives. Biochim Biophys Acta 1585: 114-125, 2002.

15. Dobrowsky RT, Kamibayashi C, Mumby MC and Hannun YA: Ceramide activates heterotrimeric protein phosphatase $2 \mathrm{~A}$. J Biol Chem 268: 15523-15530, 1993.

16. Blazquez C, Galve-Roperh I and Guzman M: De novo-synthesized ceramide signals apoptosis in astrocytes via extracellular signalregulated kinase. FASEB J 14: 2315-2322, 2000.

17. Heinrich M, Wickel M, Winoto-Morbach S, SchneiderBrachert W, Weber T, Brunner J, Saftig P, Peters C, Kronke M and Schutze S: Ceramide as an activator lipid of cathepsin D. Adv Exp Med Biol 477: 305-315, 2000.

18. Lin CF, Chen CL, Chang WT, Jan MS, Hsu LJ, Wu RH, Tang MJ, Chang WC and Lin YS: Sequential caspase-2 and caspase-8 activation upstream of mitochondria during ceramide and etoposide-induced apoptosis. J Biol Chem 279: 40755-40761, 2004.

19. Siskind LJ: Mitochondrial ceramide and the induction of apoptosis. J Bioenerg Biomembr 37: 143-153, 2005.

20. Myrick D, Blackinton D, Klostergaard J, Kouttab N, Maizel A, Wanebo and Mehta S: Paclitaxel-induced apoptosis in Jurkat, a leukemic $\mathrm{T}$ cell line, is enhanced by ceramide. Leuk Res 23: 569-578, 1999.

21. Mehta S, Blackinton D, Omar I, Kouttab N, Myrick D, Klostergaard J and Wanebo $\mathrm{H}$ : Combined cytotoxic action of paclitaxel and ceramide against the human Tu138 head and neck squamous carcinoma cell line. Cancer Chemother Pharmacol 46: 85-92, 2000.

22. Reynolds CP, Maurer BJ and Kolesnick RN: Ceramide synthesis and metabolism as a target for cancer therapy. Cancer Lett 206: 169-180, 2004.

23. Modrak DE, Gold DV and Goldenberg DM: Sphingolipid targets in cancer therapy. Mol Cancer Ther 5: 200-208, 2006.

24. Sugita M, Williams M, Dulaney JT and Moser HW: Ceramidase and ceramide synthesis in human kidney and cerebellum. Description of a new alkaline ceramidase. Biochim Biophys Acta 398: 125-131, 1975.

25. Bielawska A, Greenberg MS, Perry D, Jayadev S, Shayman JA, McKay C and Hannun YA: (1S,2R)-D-erythro-2-(N-myristoylamino)-1-phenyl-1-propanol as an inhibitor of ceramidase. J Biol Chem 271: 12646-12654, 1996.

26. Cuvillier O, Pirianov G, Kleuser B, Vanek PG, Coso OA, Gutkind S and Spiegel S: Suppression of ceramide-mediated programmed cell death by sphingosine-1-phosphate. Nature 381: 800-803, 1996.

27. Spiegel S, Cuvillier O, Edsall LC, Kohama T, Menzeleev R, Olah Z, Olivera A, Pirianov G, Thomas DM, Tu Z, van Brocklyn JR and Wang F: Sphingosine-1-phosphate in cell growth and cell death. Ann NY Acad Sci 845: 11-18, 1998.

28. Lavie Y, Cao H, Volner A, Lucci A, Han TY, Geffen V, Giuliano AE and Cabot MC: Agents that reverse multidrug resistance, tamoxifen, verapamil, and cyclosporin A, block glycosphingolipid metabolism by inhibiting ceramide glycosylation in human cancer cells. J Biol Chem 272: 1682-1687, 1997.

29. Bleicher RJ and Cabot MC: Glucosylceramide synthase and apoptosis. Biochim Biophys Acta 1585: 172-178, 2002.

30. Seelan RS, Qian C, Yokomizo A, Bostwick DG, Smith DI and Liu W: Human acid ceramidase is overexpressed but not mutated in prostate cancer. Genes Chromosomes Cancer 29: 137-146, 2000.

31. Itoh M, Kitano T, Watanabe M, Kondo T, Yabu T, Taguchi Y, Iwai K, Tashima M, Uchiyama T and Okazaki T: Possible role of ceramide as an indicator of chemoresistance: decrease of the ceramide content via activation of glucosylceramide synthase and sphingomyelin synthase in chemoresistant leukemia. Clin Cancer Res 9: 415-423, 2003.

32. Gouazé V, Yu JY, Bleicher RJ, Han TY, Liu YY, Wang H, Gottesman MM, Bitterman A, Giuliano AE and Cabot MC: Overexpression of glucosylceramide synthase and P-glycoprotein in cancer cells selected for resistance to natural product chemotherapy. Mol Cancer Res 3: 633-639, 2004.

33. Cassel DL, Hoxie JA and Cooper RA: Phorbol ester modulation of T-cell antigens in the Jurkat lymphoblastic leukemia cell line. Cancer Res 43: 4582-4586, 1983.

34. Venuat AM, Soria C, Soria J, Krief P, Mirshahi M, He L, Thomaidis A, Houllier A, Billard M and Boucheix C: High frequency of plasminogen activator secretion by malignant human lymphoid cell lines of T-cell type origin. Cancer 62: 1952-1957, 1988.
35. Matzinger P: The JAM test. A simple assay for DNA fragmentation and cell death. J Immunol Methods 145: 185-192, 1991.

36. Sun XM, Snowden RT, Skilleter DN, Dinsdale D, Ormerod MG and Cohen GM: A flow cytometric method for the separation and quantitation of normal and apoptotic thymocytes. Anal Biochem 204: 351-356, 1992.

37. Kaufmann SH, Desnoyers S, Ottaviano Y, Davidson NE and Poirier GG: Specific proteolytic cleavage of poly(ADP-ribose) polymerase: an early marker of chemotherapy-induced apoptosis. Cancer Res 53: 3976-3985, 1993.

38. Preiss J, Loomis CR, Bishop WR, Stein R, Niedel JE and Bell RM: Quantitative measurement of sn-1,2-diacylglycerols present in platelets, hepatocytes, and ras- and sis-transformed normal rat kidney cells. J Biol Chem 261: 8597-8600, 1986.

39. Shabbits JA and Mayer LD: Intracellular delivery of ceramide lipids via liposomes enhances apoptosis in vitro. Biochim Biophys Acta 1612: 98-106, 2003.

40. Mandlekar S and Kong A-NT: Mechanisms of tamoxifeninduced apoptosis. Apoptosis 6: 469-477, 2001.

41. Cabot MC, Giuliano AE, Volner A and Han TY: Tamoxifen retards glycosphingolipid metabolism in human cancer cells. FEBS Lett 394: 129-131, 1996.

42. Mader JS, Richardson A, Salsman J, Top D, De Antueno R, Duncan R and Hoskin DW: Bovine lactoferricin causes apoptosis in Jurkat T-leukemia cells by sequential permeabilization of the cell membrane and targeting of mitochondria. Exp Cell Res 313: 2634-2650, 2007.

43. Mengubas K, Riordan FA, Bravery CA, Lewin J, Owens DL, Mehta AB, Hoffbrand AV and Wickremasinghe RG: Ceramideinduced killing of normal and malignant human lymphocytes is by a non-apoptotic mechanism. Oncogene 18: 2499-2506, 1999.

44. Tiwari M, Kumar A, Sinha RA, Shrivastava A, Balapure AK, Sharma R, Bajpai VK, Mitra K, Babu S and Godbole MM: Mechanism of 4-HPR-induced apoptosis in glioma cells: evidences suggesting role of mitochondrial-mediated pathway and endoplasmic reticulum stress. Carcinogenesis 27: 2047-2058, 2006.

45. Batra S, Reynolds CP and Maurer BJ: Fenretinide cytotoxicity for Ewing's sarcoma and primitive neuroectodermal tumor cell lines is decreased by hypoxia and synergistically enhanced by ceramide modulators. Cancer Res 64: 5415-5424, 2004.

46. Maurer BJ, Melton L, Billups C, Cabot MC and Reynolds CP: Synergistic cytotoxicity in solid tumor cell lines between $\mathrm{N}$ (4-hydroxyphenyl)retinamide and modulators of ceramide metabolism. J Natl Cancer Inst 92: 1897-1909, 2000.

47. Eto M, Bennouna J, Hunter OC, Lotze MT and Amoscato AA: Importance of $\mathrm{C} 16$ ceramide accumulation during apoptosis in prostate cancer cells. Int J Urol 13: 148-156, 2006.

48. Basu S, Ma R, Mikulla B, Bradley M, Moulton C, Basu M, Banerjee S and Inokuchi J: Apoptosis of human carcinoma cells in the presence of inhibitors of glycosphingolipid biosynthesis: I. Treatment of Colo-205 and SKBR3 cells with isomers of PDMP and PPMP. Glycoconj J 20: 157-168, 2004.

49. Bruning A, Karrenbauer A, Schnabel E and Wieland FT: Brefeldin A-induced increase of sphingomyelin synthase. Assay for the action of the antibiotic in mammalian cells. J Biol Chem 267: 5052-5055, 1992.

50. Abe A and Shayman JA: Purification and characterization of 1-O-acylceramide synthase, a novel phospholipase $\mathrm{A}_{2}$ with transacylase activity. J Biol Chem 273: 8467-8474, 1998.

51. Kolesnick RN and Hemer MR: Characterization of a ceramide kinase activity from human leukemia (HL-60) cells. Separation from diacylglycerol kinase activity. J Biol Chem 265: 18803$18808,1990$.

52. Meng A, Luberto C, Meier P, Bai A, Yang X, Hannun YA and Zhou D: Sphingomyelin synthase as a potential target for D609-induced apoptosis in U937 human monocytic leukemia cells. Exp Cell Res 292: 385-392, 2004.

53. Nagarkatti $\mathrm{N}$ and Davis BA: Tamoxifen induces apoptosis in $\mathrm{Fas}^{+}$tumor cells by upregulating the expression of Fas ligand. Cancer Chemother Pharmacol 51: 284-290, 2003.

54. Kang Y, Cortina R and Perry RR: Role of c-myc in tamoxifeninduced apoptosis estrogen-independent breast cancer cells. J Natl Cancer Inst 88: 279-284, 1996.

55. Mandlekar S, Yu R, Tan TH and Kong AN: Activation of caspase-3 and c-Jun $\mathrm{NH}_{2}$-terminal kinase-1 signaling pathways in tamoxifen-induced apoptosis of human breast cancer cells. Cancer Res 60: 5995-6000, 2000. 